\title{
Solving Election Problem in Asynchronous Distributed Systems
}

\author{
SeongHoon Park \\ School of Electrical and Computer Engineering, \\ Chungbuk National Unvi. Cheongju ChungBuk 361-763, Korea \\ spark@chungbuk.ac.kr
}

\begin{abstract}
So far, the weakest failure detectors had been studied extensively for several of such fundamental problems. It is stated that Perfect Failure Detector $P$ is the weakest failure detector to solve the Election problem with any number of faulty processes. In this paper, we introduce Modal failure detector $M$ and show that to solve Election, $M$ is the weakest failure detector to solve election when the number of faulty processes is less than $\lceil n / 2\rceil$. We also show that it is strictly weaker than $P$.
\end{abstract}

\section{Introduction}

The concept of (unreliable) failure detectors was introduced by Chandra and Toueg[6], and they characterized failure detectors by two properties: completeness and accuracy. Based on the properties, they defined several failure detector classes: perfect failure detectors $\mathrm{P}$, weak failure detectors $\mathrm{W}$, eventually weak failure detectors

$\mathrm{W}$ and so on. In [6] and [8] they studied what is the "weakest" failure detector to solve Consensus. They showed that the weakest failure detector to solve Consensus with-any number of faulty processes is $\mathrm{W}$ and the one with faulty processes bounded by $\lceil\mathrm{n} / 2\rceil$ (i.e., less than $\lceil\mathrm{n} / 2\rceil$ faulty processes) is $\diamond \mathrm{W}$.

After the work of [8], several studies followed. For example, the weakest failure detector for stable leader election is the perfect failure detector P [7], and the one for Terminating Reliable Broadcast is also P [6].

In this paper, we first redefine the model of failure detectors and consider the weakest failure detectors to solve the stable leader election problem with the assumption that there is a majority of correct processes. We show that if $f$ is only bounded by a value of less than $\lceil n / 2\rceil$, where $n$ is the number of processes, the weakest failure detector to solve election is not $P$.

The rest of the paper is organized as follows. In Section 2 we describe our system model. In Section 3 we introduce the Modal Failure Detector $M$ and show that to solve Election, $M$ is necessary while $P$ is not, whereas $M$ is sufficient when a majority of the processes are correct. Finally, Section 4 summarizes the main contributions of this paper and discusses related and future work. 


\section{Model and Definitions}

Our model of asynchronous computation with failure detection is the one described in [5]. In the following, we only discuss some informal definitions and results that are needed for this paper.

\subsection{Processes}

We consider a distributed system composed of a finite set of processes $\Omega=\{1,2, \ldots, n\}$ to be completely connected through a set of channels. Each process has a unique id and its priority is decided based on the id, i.e., a process with the lowest id has the highest priority. Communication is by message passing, asynchronous and reliable. Processes fail by crashing and the crashed process does not recover. We consider systems where at least one process is correct (i.e. $f<|\Omega|$ ).

A failure detector is a distributed oracle which gives hints on failed processes. We consider algorithms that use failure detectors. An algorithm defines a set of runs, and a run of algorithm $A$ using a failure detector $D$ is a tuple $R=\langle F, H, I, S, T>$ : $I$ is an initial configuration of $A ; S$ is an infinite sequence of events of $A$ (made of process histories); $T=\mathrm{t}_{0} \cdot \mathrm{t}_{1} \cdot \mathrm{t}_{2} \cdots \mathrm{t}_{\mathrm{k}}$ is a list of increasing time values indicating when each event in $S$ occurred where $\mathrm{t}_{0}$ denotes a starting time; $F$ is a failure pattern that denotes the set $F(t)$ of processes that have crashed through any time $t$. A failure pattern is a function $F$ from $T$ to $2^{\varrho}$. The set of correct processes in a failure pattern $F$ is noted $\operatorname{correct}(F)$ and the set of incorrect processes in a failure pattern $F$ is noted $\operatorname{crashed}(F)$; $H$ is a failure detector history, which gives each process $p$ and at any time $t$, a (possibly false ) view $H(p, t)$ of the failure pattern. $H(p, t)$ denotes a set of processes, and $q \in H(p, t)$ means that process $p$ suspects process $q$ at time $t$.

\subsection{Failure Detector Classes, Reducibility and Transformation}

Two completeness properties have been identified. Strong Completeness, i.e. there is a time after which every process that crashes is permanently suspected by every correct process, and Weak Completeness, i.e. there is a time after which every process that crashes is permanently suspected by some correct process. Four accuracy properties have been identified. Strong Accuracy, i.e. no process is never suspected before it crashes. Weak Accuracy, i.e. some correct process is never suspected. Eventual Strong Accuracy ( $\diamond$ Strong), i.e. there is a time after which correct processes are not suspected by any correct process; and Eventual Weak Accuracy ( $\diamond$ Weak), i.e. there is a time after which some correct process is never suspected by any correct process.

The notation of problem reduction was first introduced in the problem complexity theory [10], and in the formal language theory [9]. It has been also used in the distributed computing [11,12]. We consider the following definition of problem reduction. An algorithm $A$ solves a problem $B$ if every run of $A$ satisfies the specification of $B$. A problem $B$ is said to be solvable with a class $C$ if there is an algorithm which solves $B$ using any failure detector of $C$. A problem $B_{l}$ is said to be 
reducible to a problem $B_{2}$ with class $C$, if any algorithm that solves $B_{2}$ with $C$ can be transformed to solve $B_{1}$ with $C$. If $B_{l}$ is not reducible to $B_{2}$, we say that $\mathrm{B}_{l}$ is harder than $B_{2}$.

\subsection{The Stable Leader Election}

The stable leader election problem is described as follows: at any time, at most one process considers itself the leader, and at any time, if there is no leader, a leader is eventually elected. Once a process is elected to be a leader, it can't be demoted before crash. The stable leader election problem is specified by the following two properties:

- Safety: At any time, if a correct process has its leader set to true, then all other processes that had their leader set to true crashed.

- Liveness: At any time, there eventually exists a process that has its leader set to true.

\section{Failure Detector to Solve Election}

We define the Modal failure detector $M$, which is weaker than $P$. We show that, to solve Election: (1) $M$ is necessary (for any environment), and (2) $M$ is sufficient for any environment with a majority of correct processes. We then show that (3) $P$ is strictly stronger than $M$ for any environment where at least one processes can crash in a system of at least three processes.

\subsection{Modal Failure Detector}

Each module of failure detector $M$ outputs a subset of the range $2^{\varrho}$. The most important property of $M$, denoted by Modal Accuracy, is that a process that was once confirmed to be correct is not suspected before crash. Let $H_{M}$ be any history of such a failure detector $M$. Then $M$ satisfies the following properties:

- Strong Completeness: There is a time after which every process that crashes is permanently suspected by every correct process.

- Eventual Weak Accuracy: There is a time after which some correct process is never suspected by any correct process.

- Modal Accuracy: Initially, every process is suspected. After that, any process that is once confirmed to be correct is not suspected before crash. More precisely:

\subsection{The Necessary Condition for Election}

The basic idea of our algorithm is the following. Initially, the value of $F L_{i}$ and $C L_{i}$ is set to $\Omega$ and $\Phi$ respectively. That means that initially every process is suspected and none is confirmed. After that each process periodically invokes election and waits until the result of election is returned. If the received leader is in $F L_{i}$, then process $i$ removes it from $F L_{i}$ and puts it into $C L_{i}$. If it is not identical with the current leader then process $i$ puts the id of the current leader into $F L_{i}$ since the leader that was once confirmed to be correct has crashed. 


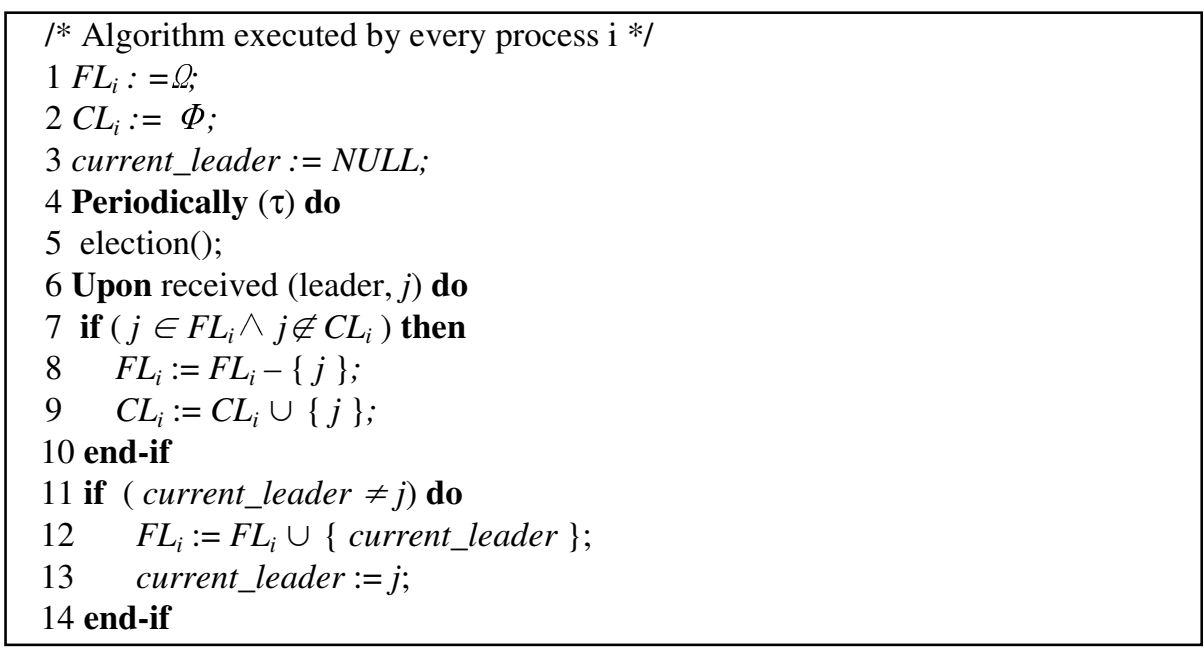

Fig. 1. Emulating $M$ using Election

\section{Lemma 3.1. The algorithm of Fig. 2 uses Election to implement $M$.}

Proof. We show below that $F L_{i}$ satisfies Strong Completeness, Eventually Weak Accuracy and Modal Accuracy properties of $M$.

- Strong Completeness: Once elected as a leader, the process can be demoted only if it crashes. Initially, every process is suspected by invoking $F L_{i}:=\Omega$ n line 2 of fig. 1. Therefore, it satisfies strong completeness. After that the correct process $i$ removes $j$ from $F L_{i}$ in line 8 of fig. 1 only once at most and only if process $i$ received $j$ as a leader. Let assume that process $j$ is elected as the leader and then crashes at time $t$, and let assume that process $i$ is a correct process. Then by the liveness property of election, process $i$ eventually receives the message (leader, j). Assume by contradiction that strong completeness is violated. It implies that process $i$ never puts $j$ into $F L_{i}$ even though it has crashed. This means that process $i$ invokes election in line 5 , but always receive $j$ as a leader in line 6 of fig.1, even though it has crashed. However, because leader process $j$ crashes at time $t$, there is a time $t^{\prime}$ so that for every $t^{\prime}$ '> $t$ ', process $i$ never receives process $j$ as a leader by the liveness property of election: a contradiction.

- Eventually Weak Accuracy: By contradiction, assume that eventual weak accuracy is violated. It implies that with every correct process $j$, there is a correct process $i$ that suspects it. Let process $j$ be elected as a leader and it doesn't crash. That is to hold, there should be a correct process $i$ that never stops suspecting $j$ even though $j$ is elected to be the leader in the algorithm of fig.1. However, by the liveness property of the election algorithm of fig. 1, once correct processes $j$ is elected as a leader and doesn't crash, then every correct process eventually receives the message (leader, $j$ ) and knows that $j$ is a leader: contradiction. 
- Modal Accuracy: By contradiction, assume that modal accuracy is violated. By algorithm fig. 1, the predicate $j \notin F L_{i}(t)$ implies that at time $t^{\prime \prime}<t$, process $j$ is elected and removed from $F L_{i}$. The predicate $j \in F L_{i}\left(t^{\prime}\right)$ implies that at time $t^{\prime}>t$, process $k$ ( $k \neq j$ ) is elected as a leader when $j$ is the current leader and $j$ is inserted to $F L_{i}$. Given that a process was once elected as a leader in stable election, the process can be demoted only if it crashes. Thus, the new leader can be returned only if the current leader crashes. That implies $j \in F\left(t^{\prime}\right)$. So it is a contradiction.

The following theorem follows directly from Lemma 3.1.

Theorem 3.1. If any failure detector $\mathrm{D}$ solves election, then $\mathrm{M} \mu \mathrm{D}$.

\subsection{The Sufficient Condition for Election}

Periodically processes wait for an output from $M$ to ensure the leader's crash. If the process receives from $M$ the information that the current leader has crashed and at the same time the status of current leader is not false, i.e., (current_leader ${ }_{i} \neq \perp$ ), the process invokes consensus with a new candidate for leader and decides the new leader returned by consensus. Otherwise the process decides the current leader. We assume that every process $i$, either crashes, or invokes election in Fig.2. The new leader candidate of participant $i$, denoted new_candidate ${ }_{i}$, is decided by the next function. The current leader, denoted by current_leader ${ }_{i}$, is decided by the consensus function. The status of participant $i$ whether it is a leader or not is decided by the variable, leader $_{i}$. That is, if the variable leader $_{i}$ is set true, the process $i$ considers itself a leader.

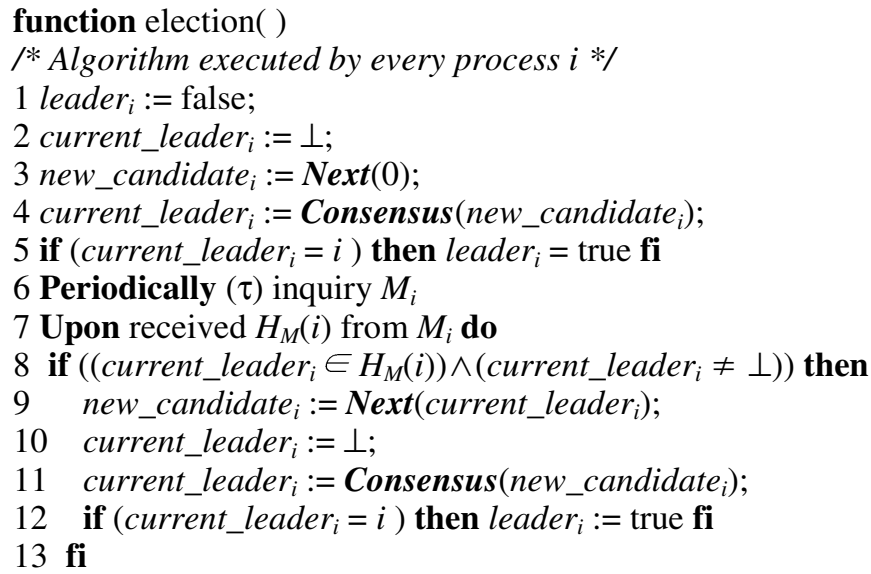

Fig. 2. Transforming Consensus into Election with $M$

We define the Next function of process $i$ in Fig.2 as follows.

$$
\operatorname{Next}(k)=\min \{j \mid j \notin H(i, t) \wedge j \neq k\} .
$$

Lemma 3.2. The algorithm of Fig. 2 uses $M$ to transform Consensus into Election.

Proof. We consider the properties of Election separately. 
- Safety: A process that every correct process does not suspect is eventually elected as a leader by Next and Consensus functions. Let process $i$ be the current leader elected at time $t$ that is denoted by current_leader $(t)=i$, then clearly the process $i$ is a correct process that the failure detector $M$ of every correct process does not suspect at time $t^{\prime}, t^{\prime}<t$. By Modal Accuracy the new leader is elected only when the current leader $i$ has crashed.

- Liveness: Consider leader $i$ that is elected at time $t$ in Fig.3. After that, if the leader process crashes at time $t^{\prime}, t^{\prime}>t$, then by Strong Completeness of $M$, there is a time after that some correct processes suspect the current leader. There is eventually some correct process which executes line 7-11 of fig. 3. They decide a prospective leader by using the Next function and transfer it as a parameter to Consensus function. With the Validity property of Consensus, a process decides its leader only if some process has invoked consensus. By the Termination property of Consensus, every correct process eventually decides a leader that ensures the Liveness property of Election.

We define here failure detector $M$. Each module of $M$ outputs a subset of $\Omega$. Failure detector $M$ satisfies Strong Completeness and Eventually Weak Accuracy, together with Modal Accuracy. Since Consensus is solvable with Strong Completeness and Eventually Weak Accuracy for any environment with a majority of correct processes [8], then the following theorem follows from Lemma 3.2:

Theorem 3.2. $M$ solves Election for any environment where a majority of processes are correct, $f<n / 2$.

Finally, we can state the following theorem from Theorem 3.1 and Theorem 3.2.

Theorem 3.3. For any environment with $f<n / 2, M$ is the weakest failure detector to solve Election.

Proof : It is straightforward from Theorem 3.1 and Theorem 3.2

\subsection{Modal Failure Perfection Is Not Perfection}

Obviously, failure detector $P$ can be used to emulate $M$ for any environment, i.e., $M \mu$ $P$. We state in the following that the converse is not true for any environment where at least one processes can crash in a system.

Theorem 3.4. $P \sim M$ for any environment where at least one process can crash in a system.

Proof. (By contradiction). We assume that there is an algorithm $A_{M \rightarrow P}$ that transforms $M$ into failure detector $P$. Then we show the fact that $P$, transformed by above the algorithm, satisfies Strong Completeness, but it does not satisfy Strong Accuracy: So it is a contradiction. We denote by $\operatorname{output}(P)$ the variable used by $A_{M \rightarrow P}$ to emulate failure detector $P$, i.e., output $(P)_{i}$ denotes the value of that variable at a given process $i$. Let $F_{1}$ be the failure pattern where process 1 has initially crashed and no other process has crashed, i.e., $F_{1}\left(t_{0}\right)=\{1\}$. Let $H_{1}$ be the failure detector history where all 
processes permanently output $\{1\}$ at $t^{\prime}, t^{\prime}>t_{0}$; i.e., $\forall i \in \Omega, \exists t^{\prime} \in T, t^{\prime}>t_{0}: H_{l}\left(i, t^{\prime}\right)=$ $\{1\}$. Clearly, $H$ belongs to $M\left(F_{1}\right)$. Since variable output $(P)$ satisfies Strong Completeness of $P$ then there is a partial run of $A_{M \rightarrow P}, R_{1}=\left\langle F_{1}, H_{1}, I, S_{1}, T\right\rangle$ such that $\exists j \in \Omega, \exists t t^{\prime} \in T, t^{\prime \prime} \geq t$ ' $:\{1\} \subset \operatorname{output}(P)_{j}$. Consider failure pattern $F_{2}$ such that $\operatorname{correct}\left(F_{2}\right)=\Omega\left(F_{2}\right.$ is failure free $)$ and define the failure detector history $H_{2}$ such that $\forall$ $i \in \Omega, \forall t \in T: H_{2}(i, t)=\{1\}, t^{\prime} \leq t \leq t^{\prime \prime}$ and $H_{2}(i, t)=\Phi, t>t^{\prime \prime}$. Note that $H_{2} \in$ $M\left(F_{2}\right)$ and $t^{\prime} \leq t \leq t^{\prime}, \quad \forall i \in \Omega-\{1\}: H_{l}(i, t)=H_{2}(i, t)$. Consider $R_{2}=<F_{2}, H_{2}, I, S_{2}, T$ $>$ of $A_{M \rightarrow P}$ such that $S_{1}[k]=S_{2}[k], \forall t \in T, t^{\prime} \leq t \leq t^{\prime}$ '. Let $R_{2}$ outputs a history $H_{P} \in$ $P\left(F_{2}\right)$. Since partial runs of $R_{1}$ and $R_{2}$ for $t^{\prime} \leq t \leq t^{\prime \prime}$ are identical, the resulting history $H_{P}$ of process $j$ is: $\forall t \in T, t^{\prime} \leq t \leq t^{\prime \prime}:\{1\} \subset$ output $(P)_{j}$. But in $R_{2}$, at $t, t^{\prime} \leq t \leq t^{\prime \prime}: 1$ $\in \operatorname{output}(P)_{j}$ and $l \in \operatorname{correct}\left(F_{2}\right)$, which means that $P$ violates Strong Accuracy: a contradiction.

\section{Concluding Remarks}

So far the applicability of these results to problems other than Consensus has been discussed in $[6,13,14,15]$. In [8], it is shown that Consensus is sometimes solvable where Election is not. In [7], it was shown that the weakest failure detector for Election is Perfect failure detector $P$, if we consider Election to be defined among every pair of processes. If we consider however Election to be defined among a set of at least three processes and at most one can crash, this paper shows that $P$ is not necessary for Election. An interesting consequence of this result is that there exists a failure detector that is weaker than Perfect failure detector $P$ to solve Election at the environment where a majority of processes are correct, $f<n / 2$.

This paper introduces Modal failure detector $M$ which is weaker than Perfect failure detector $P$, and shows that: (1) $M$ is necessary to solve Election, (2) $M$ is sufficient to solve Election, and (3) $M$ is the weakest failure detector to solve Election when a majority of the processes are correct. A corollary of our results above is that we can construct a failure detector that is strictly weaker than $P$, and yet solves Election.

Is this only theoretically interesting? We believe not, as we will discuss below. Interestingly, failure detector $M$ consists of $\diamond S+$ Modal Accuracy and it helps deconstruct Election: intuitively, $\diamond S$ conveys the pure agreement part of Election, whereas Modal Accuracy conveys the specific nature of detecting a leader crash. Besides better understanding the problem, this deconstruction provides some practical insights about how to adjust failure detector values in election protocols.

In terms of the practical distributed applications, we can induce some interesting results from the very structure of $\diamond S+$ Modal Accuracy on the solvability of Election. In real distributed systems, failure detectors are typically approximated using timeouts. To implement the Modal Accuracy property, one needs to choose a large timeout value in order to reduce false leader failure suspicions. However, to implement $\diamond S$, a time-out value that is not larger than the one for Modal Accuracy is needed. Therefore an election algorithm based on $\diamond S+$ Modal Accuracy might reduce possibility of violating the safety condition but speed up the consensus of electing a new leader in the case of a leader crash. 


\section{References}

1. G. LeLann: Distributed Systems-towards a Formal Approach. Information Processing 77, B. Gilchrist, Ed. North-Holland, 1977.

2. H. Garcia-Molina: Elections in a Distributed Computing System. IEEE Transactions on Computers, C-31 (1982) 49-59

3. H. Abu-Amara and J. Lokre: Election in Asynchronous Complete Networks with Intermittent Link Failures. IEEE Transactions on Computers, 43 (1994) 778-788

4. G. Singh: Leader Election in the Presence of Link Failures. IEEE Transactions on Parallel and Distributed Systems, 7 (1996) 231-236

5. M. Fischer, N. Lynch, and M. Paterson: Impossibility of Distributed Consensus with One Faulty Process. Journal of ACM, (32) 1985 374-382

6. T. Chandra and S.Toueg: Unreliable Failure Detectors for Reliable Distributed Systems. Journal of ACM, 43 (1996) 225-267

7. L. Sabel and K. Marzullo. Election Vs. Consensus in Asynchronous Distributed Systems. In Technical Report Cornell Univ., Oct. 1995

8. T. Chandra, V. Hadzilacos and S. Toueg: The Weakest Failure Detector for Solving Consensus. Journal of ACM, 43 (1996) 685-722

9. J. E. Hopcroft and J. D. Ullman: Introduction to Automata Theory, Languages and Computation. Addison Wesley, Reading, Mass., 1979

10. Garey M.R. and Johnson D.S: Computers and Intractability: A Guide to the Theory of NPCompleteness. Freeman W.H \& Co, New York, 1979

11. Eddy Fromentin, Michel RAY and Frederic TRONEL: On Classes of Problems in Asynchronous Distributed Systems. In Proceedings of Distributed Computing Conference. IEEE, June 1999

12. Hadzilacos V. and Toueg S: Reliable Broadcast and Related Problems. Distributed Systems (Second Edition), ACM Press, New York, pp.97-145, 1993

13. R. Guerraoui: Indulgent Algorithms. In: Proceedings of the ACM Symposium on Principles of Distributed Computing, New York: ACM Press 2000

14. Schiper and A. Sandoz: Primary Partition: Virtually-Synchronous Communication harder than Consensus. In Proceedings of the 8th Workshop on Distributed Algorithms, 1994

15. R. Guerraoui and A. Schiper: Transaction model vs. Virtual Synchrony model: bridging the gap. In: K. Birman, F. Mattern and A. Schiper (eds.): Distributed Systems: From Theory to Practice. Lecture Notes in Computer Science, Vol. 938. Springer- Verlag, Berlin Heidelberg New York (1995) 121-132 